\title{
Sequence variant analysis reveals poor correlations in microbial taxonomic abundance between humans and mice after gnotobiotic transfer
}

\author{
Farnaz Fouladi ${ }^{1}$ Elaine M. Glenny ${ }^{2} \cdot$ Emily C. Bulik-Sullivan $^{2} \cdot$ Matthew C. B. Tsilimigras $\mathbb{D}^{1,2} \cdot$ Michael Sioda $^{1}$. \\ Stephanie A. Thomas ${ }^{2} \cdot$ Yunfei Wang $^{3} \cdot$ Zorka Djukic $^{2} \cdot$ Quyen Tang $^{2} \cdot$ Lisa M. Tarantino ${ }^{4,5} \cdot$ Cynthia M. Bulik $^{2,6,7}$. \\ Anthony A. Fodor ${ }^{1} \cdot$ Ian M. Carroll ${ }^{2,8}$
}

Received: 12 September 2019 / Revised: 20 March 2020 / Accepted: 24 March 2020 / Published online: 20 April 2020

(c) The Author(s), under exclusive licence to International Society for Microbial Ecology 2020

\begin{abstract}
Transplanting human gut microbiotas into germ-free (GF) mice is a popular approach to disentangle cause-and-effect relationships between enteric microbes and disease. Algorithm development has enabled sequence variant (SV) identification from 16S rRNA gene sequence data. SV analyses can identify which donor taxa colonize recipient GF mice, and how SV abundance in humans is replicated in these mice. Fecal microbiotas from 8 human subjects were used to generate 77 slurries, which were transplanted into $153 \mathrm{GF}$ mice. Strong correlations between fecal and slurry microbial communities were observed; however, only $42.15 \pm 9.95 \%$ of SVs successfully transferred from the donor to the corresponding recipient mouse. Firmicutes had a particularly low transfer rate and SV abundance was poorly correlated between donor and recipient pairs. Our study confirms human fecal microbiotas colonize formerly GF mice, but the engrafted community only partially resembles the input human communities. Our findings emphasize the importance of reporting a standardized transfer rate and merit the exploration of other animal models or in silico tools to understand the relationships between human gut microbiotas and disease.
\end{abstract}

\section{Introduction}

The gut microbiota's role in human health and energy balance has been widely investigated since it was posited that this

These authors contributed equally: Farnaz Fouladi, Elaine M. Glenny

Supplementary information The online version of this article (https:// doi.org/10.1038/s41396-020-0645-z) contains supplementary material, which is available to authorized users.

$\triangle$ Anthony A. Fodor

afodor@uncc.edu

$凶$ Ian M. Carroll

ian_carroll@med.unc.edu

1 Department of Bioinformatics and Genomics, University of North Carolina at Charlotte, Charlotte, NC 28223, USA

2 Department of Nutrition, University of North Carolina at Chapel Hill, Chapel Hill, NC 27599, USA

3 Department of Psychology and Neuroscience, University of North Carolina at Chapel Hill, Chapel Hill, NC 27599, USA complex microbial community influences body fat regulation [1]. Elucidating the functional role of gut microbial communities in the initiation and maintenance of disease is a critical first step towards developing novel, effective therapies for gut microbiota-associated diseases. Although numerous associations between altered enteric microbial community compositions and human diseases have been reported [2-8], it has been challenging to demonstrate specific disease-associated enteric microbes as etiological agents. An attractive approach for determining causal relationships between the intestinal

4 Department of Genetics, University of North Carolina at Chapel Hill, Chapel Hill, NC 27599, USA

5 Division of Pharmacotherapy and Experimental Therapeutics, University of North Carolina at Chapel Hill, Chapel Hill, NC 27599, USA

6 Department of Psychiatry, University of North Carolina at Chapel Hill, Chapel Hill, NC 27599, USA

7 Department of Medical Epidemiology and Biostatistics, Karolinska Institutet, Stockholm, Sweden

8 Center for Gastrointestinal Biology and Disease, University of North Carolina at Chapel Hill, Chapel Hill, NC 27599, USA 
microbiota and specific diseases is to transfer viable human fecal microbial communities into germ-free (GF) mice (mice born and living in the absence of microorganisms). Seminal studies have demonstrated that transplanting fecal microbiotas from obese individuals into GF mice results in increased mouse adiposity compared to GF mice colonized with fecal microbiotas from lean individuals [9, 10]. Multiple groups have also demonstrated that transplantation of fecal microbial communities from humans into GF or antibiotic-treated mice transmits specific phenotypes or metabolic profiles [9-14].

Investigations using the gnotobiotic approach have reproducibly demonstrated that the transplanted human fecal microbiotas differ from those of specific pathogen-free (SPF) mice $[11,15,16]$. However, these studies and others have also reported that the enteric microbial community in recipient mice is substantially different from that of the human donors $[11,12,15,16]$. Depending on the taxonomic level and the computational methods used to measure similarity, $20-88 \%$ of the human donor gut microbiota has been reported to colonize the gastrointestinal (GI) tract of GF or antibiotic-treated rodents after transplantation $[11,12,15-18]$. Multiple factors likely contribute to this extensive variation of colonization, including strong selection pressure from the host (genetic effect) [19, 20], cage and isolator effects $[12,21,22]$, and different technical approaches to fecal transplantation [23].

Recent improvements in bioinformatic pipelines have increased the resolution of variant classification that can be reliably generated from 16S rRNA gene sequence data [24-26]. Utilizing these methods, it is possible to resolve sequence variants (SVs) to separate exact $16 \mathrm{~S}$ rRNA gene sequences from sequencing errors. In the current study, we sought to utilize these recent advances in algorithm development to achieve a high-resolution view of microbial colonization dynamics and address important questions regarding human fecal transfer into GF mice. First, we calculated the transfer efficiency of the original donor microbial community into GF mice by measuring the presence of enteric microbial communities in (i) human fecal samples, (ii) slurries used for colonization, and (iii) mouse fecal pellets. Moreover, the large number of recipient male and female GF mice colonized with each donor sample allowed us to consider not only the proportion of taxa shared between human donors and mouse recipients, but also to directly compare the relative abundance of each taxon between paired human donors and mouse recipients over the course of 28 days following colonization. In addition, as the relative abundance of taxa is influenced by environmental factors (e.g., diet) [27] we colonized GF mice with fecal samples from patients with anorexia nervosa (AN) at two-time points and analyzed mouse fecal microbiotas at multiple time points. Our study, therefore, presents a detailed picture of how human fecal microbial communities longitudinally change when they are introduced to the mouse gut ecosystem.

\section{Materials and methods}

\section{Ethics approval}

Human fecal sample collection was approved by the Biomedical Institutional Review Board of the University of North Carolina (UNC) at Chapel Hill. All human participants provided written consent before study participation. All animal studies were approved by the Institutional Animal Care and Use Committee of UNC-Chapel Hill.

\section{Human study participants}

Adult female patients with AN $(n=4)$ and age-, race-, and sex-matched healthy controls $(n=4)$ consented to participate in this study. Patients met DSM-5 criteria for AN [28] and were recruited as inpatients from the Center of Excellence for Eating Disorders at UNC-Chapel Hill. Healthy controls were recruited using university flyers and listservs and had no history of either a body mass index outside $18.5-24.9 \mathrm{~kg} / \mathrm{m}^{2}$ or an eating disorder. Exclusion criteria were applied as previously described [29]. These criteria focused on factors known to influence the composition of the gut microbiota including: (i) history of GI tract surgery (other than cholecystectomy); (ii) history of inflammatory bowel diseases, irritable bowel syndrome, celiac disease, or any other diagnosis that could explain chronic or recurring bowel symptoms; (iii) treatment in the previous 2 months with antibiotics, nonsteroidal anti-inflammatory drugs, or steroids; and (iv) intentional use of probiotics in the previous 2 months.

\section{Human fecal sample collection}

Patients with AN provided a fecal sample at time of admission to the eating disorders unit (T1) and at time of discharge after weight restoration (T2). Once a patient had collected a fecal sample it was placed at $4{ }^{\circ} \mathrm{C}$ until it could be transported to the laboratory. Healthy controls provided a single fecal sample. Healthy controls collected each sample at home using a stool collection kit as previously described [30] and stored at $4{ }^{\circ} \mathrm{C}$ until they were shipped overnight with ice packs to our laboratory. Fresh fecal samples arrived at the laboratory on ice and were immediately mechanically homogenized with a spatula in a biological safety cabinet under aerobic conditions, aliquoted into 2-mL cryovials without any added 
cryoprotectants and stored at $-80^{\circ} \mathrm{C}$ until needed for transplantation into GF mice.

\section{Transplant of human fecal microbiotas into GF mice}

Sterile Dulbecco's phosphate-buffered saline (PBS) was prereduced by placing in an anaerobic chamber overnight to remove oxygen. $10 \%(\mathrm{w} / \mathrm{v})$ of freshly thawed human feces was suspended in prereduced PBS under anaerobic conditions and fecal slurries were vortexed for 5 min [18]. Suspended bacteria were separated from fibrous material either by centrifugation at $1000 \times g$ for $3 \mathrm{~min}$ or $9 \times g$ for $3 \mathrm{~min}$, or by filtration through a sterile $100 \mu \mathrm{m}$ filter. The resulting slurries were aliquoted $(300 \mu \mathrm{L})$ into individual cryotubes under anaerobic conditions. 8-9-week-old adult male and female C57BL/6 GF mice were randomized into blinded groups and orally gavaged once with $10 \mathrm{~mL} / \mathrm{kg}(250 \mu \mathrm{L}$ maximum) slurry. As the efficiency of engraftment of microbiotas into male or female mice was uncertain, both sexes were used in our study. To avoid cage effects on the intestinal microbiota, mice were singly housed in individually ventilated cages with ad libitum access to autoclaved water and rodent chow. Mice were placed into clean cages with autoclaved bedding, hut, nestlet, water, and food every 14 days. Mice were handled under sterile flow hoods and animal handler's gloves were disinfected with Virkon S between mice to collect fresh fecal pellets, body weights, and food consumption every 7 days. 28 days after colonization, mice were euthanized with $\mathrm{CO}_{2}$ followed by cervical dislocation.

\section{Microbiota analysis}

Genomic microbial DNA from human fecal samples, slurries, and mouse fecal pellets was isolated via a phenolchloroform extraction method combined with a beadbeating step using $0.1 \mathrm{~mm}$ glass beads (Bio Spec products, Bartlesville, OK) to physically disrupt bacterial cells and a DNA clean-up kit (Qiagen DNeasy Blood and Tissue extraction kit, Valencia, CA) as previously described [31]. Fecal microbiotas and slurries were characterized by creating sequencing libraries from the variable 4 (V4) region of the 16S rRNA gene (515-806 bp) using polymerase chain reaction (PCR) and sequencing on the Illumina MiSeq platform (Illumina, San Diego, CA) at the High-Throughput Sequencing Facility in the Carolina Center for Genome Sciences at the UNC School of Medicine as previously described [32]. Mock microbial communities (MMCs, ZymoBIOMICS ${ }^{\mathrm{TM}}$, Tustin, CA) with known bacterial DNA were used as a defined reference for PCR and sequencing to detect potential contaminants. MMCs included genomic DNA from
Pseudomonas aeruginosa, Escherichia coli, Salmonella enterica, Lactobacillus fermentum, Enterococcus faecalis, Staphylococcus aureus, Listeria monocytogenes, and Bacillus subtilis. For quality control, PhiX was spiked into Illumina MiSeq runs at a range of $6.62-15.56 \%$ (reads that matched PhiX genome were removed using filterAndTrim function in DADA2). Q30 for all runs was $100 \%$ with cluster density ranging between 609 and 1,296 per $\mathrm{mm}^{2}$ and a passing filter ranging between $82.9-96.2 \%$. Sequence reads were demultiplexed using an automatic bioinformatics pipeline "BioLockJ" (https:// github.com/msioda/BioLockJ). Mock communities were found close to their expected concentrations and genera not expected to be present were found at low relative abundance (Supplementary Fig. 1). Forward reads were used for the downstream analyses. Primers from the forward reads were removed by custom Java code. The DADA2 pipeline was used to generate SVs at $100 \%$ identity threshold. Sequences were truncated to 200 bases. Sequences with Ns and with expected errors higher than 2 were discarded using filterAndTrim function in DADA2. After removing 1 human fecal sample, 1 slurry, and 7 mouse fecal pellets that did not meet sequence quality, the final number of reads was 41,090,870 $(59,208.75 \pm$ 32,557.47 per sample) and total number of SVs generated by DADA2 was 2,157 . Read counts were normalized using the following formula:

$\log _{10}\left(\left[\frac{\text { Raw count in sample }(i)}{\# \text { of sequences in sample }(i)} \times\right.\right.$ average \# of sequences per sample $\left.]+1\right)$

SVs that were present in fewer than $10 \%$ of samples were removed prior to any statistical analyses, resulting in the final number of 279 SVs. Taxonomic classification was performed using DADA2 formatted reference databases (silva_nr_v128_train_set.fa.gz and silva_species_assignment_v128.fa.gz) and assignTaxonom and addSpecies functions in DADA2. All sequences have been deposited in NCBI Sequence Read Archive (PRJNA558494), and all scripts are available at https:// github.com/FarnazFouladi/MicrobiotaTransfer.

\section{Rate of SV transfer from humans to GF mice}

In order to determine the rate of SV transfer from human donors to GF mice during the colonization process, we compared the presence of SVs between (i) the human fecal samples and their corresponding slurries; and (ii) the slurries and their corresponding fecal pellets from the recipient GF mice. To first determine the probabilities (frequencies) of each SV being shared between both human fecal samples and slurries, being detected only in human fecal samples, or only being detected in slurries, we used the 
following formulas:

-shared

number of times that a SV was observed in a human fecal sample and its paired slurry final number of human fecal sample - slurry pairs $(n=63)$

p-only in human feces

number of times that a SV was observed in a human fecal sample but not in its paired slurry final number of human fecal sample - slurry pairs $(n=63)$

$p$-only in slurries

number of times that a SV was observed in a slurry but not in its paired human fecal sample final number of human fecal sample - slurry pairs $(n=63)$

Similarly, the probabilities of each SV being shared between slurries and mouse fecal pellets, only being detected in slurries, or only being detected in mouse fecal pellets were determined using the following formulas:

p-shared

number of times that a SV was observed in a slurry and its paired mouse fecal pellet final number of slurry - mouse fecal pellet pairs $(n=135)$

p-only in slurries

number of times that a SV was observed in a slurry but not in its paired mouse fecal pellet final number of slurry - mouse fecal pellet pairs $(n=135)$

p-only in mouse fecal pellets

number of times that a SV was observed in a mouse fecal pellet but notin its paired slurry final number of slurry - mouse fecal pellet pairs $(n=135)$

\section{Statistical tests}

A nonparametric Spearman's rank-order correlation was used to study the relationship between SVs in human fecal samples, slurries, and mouse fecal pellets. All $p$ values from Spearman correlations were corrected for multiple hypothesis testing using the Benjamini-Hochberg procedure with a threshold false discovery rate (FDR) of 0.05 . The ADONIS test, a permutation-based multivariate ANOVA using Bray-Curtis dissimilarity matrices, was used to compare the fecal microbiota composition between samples. One-way ANOVA with a post-hoc Tukey's Honestly Significant Difference (HSD) test was used to compare the transfer efficiency of SVs between different taxonomic groups in recipient GF mice. Adjusted $p$ values less than 0.05 were considered significant.

\section{Results}

This study used 12 human donor fecal samples for colonization experiments (4 healthy controls and 4 patients with AN before and after clinical renourishment). For each human donor, 3-7 slurries were prepared for independent colonization experiments. While we used both $100 \mu \mathrm{M}$ cell strainers and centrifugation to remove fecal debris, our analyses demonstrate that preparation method did not have a significant effect on transfer efficiency of microbes from slurries into recipient GF mice (Supplementary Fig. 2). For each freshly prepared slurry, 1-4 C57BL/6 GF mice were colonized. In sum, this study used 12 fecal samples provided by 8 human donors for colonization of 153 GF mice using 77 different slurries (Table 1A, B).

Fecal pellets were collected from mice once every 7 days following colonization and mice were followed for 28 days (4 time points per mouse). Based on Bray-Curtis dissimilarity matrices, we observed distinct clustering between mouse and human samples, with human fecal samples and slurries clustering together (ADONIS test, $r^{2}=0.11$, $p$ value $=0.001$; Fig. 1a). Although forward reads were used for analysis, paired reads generated comparable data (Supplementary Fig. 3a). The transplanted microbial community was stable over the 4-week colonization period
Table 1 Number of biological samples generated, collected, and analyzed in this study. A) Number of human slurries generated and mouse fecal samples collected. B) Number of human slurries and mouse fecal samples analyzed after removing samples that were not sequenced or failed to meet sequencing quality thresholds.

\begin{tabular}{l}
$\begin{array}{l}\text { Human participants } \\
\text { Timepoint }\end{array}$ \\
\hline
\end{tabular}

A. Number of samples collected in the study

$\begin{array}{llllllllllllll}\text { Slurries } & 6 & 7 & 6 & 6 & 6 & 6 & 7 & 8 & 6 & 6 & 7 & 6 & 77\end{array}$

$\begin{array}{llllllllllllll}\text { Recipient mice } & 12 & 14 & 12 & 12 & 12 & 12 & 14 & 17 & 12 & 12 & 12 & 12 & \mathbf{1 5 3}\end{array}$

$\begin{array}{lllllllllllllll}\text { Mouse fecal pellets (4 timepoints) } & 48 & 56 & 48 & 48 & 48 & 48 & 56 & 68 & 48 & 48 & 48 & 48 & \mathbf{6 1 2}\end{array}$

B. Number of samples analyzed after removing samples that failed to meet sequencing quality thresholds

$\begin{array}{llllllllllllll}\text { Slurries } & 6 & 3 & 6 & 6 & 6 & 6 & 5 & 5 & 6 & 6 & 7 & 6 & \mathbf{6 8}\end{array}$

$\begin{array}{llllllllllllll}\text { Recipient mice } & 12 & 14 & 12 & 12 & 12 & 12 & 14 & 17 & 12 & 12 & 12 & 12 & \mathbf{1 5 3}\end{array}$

$\begin{array}{llllllllllllll}\text { Mouse fecal pellets (4 timepoints) } & 47 & 55 & 48 & 48 & 48 & 48 & 56 & 66 & 47 & 48 & 48 & 46 & \mathbf{6 0 5}\end{array}$ 
A

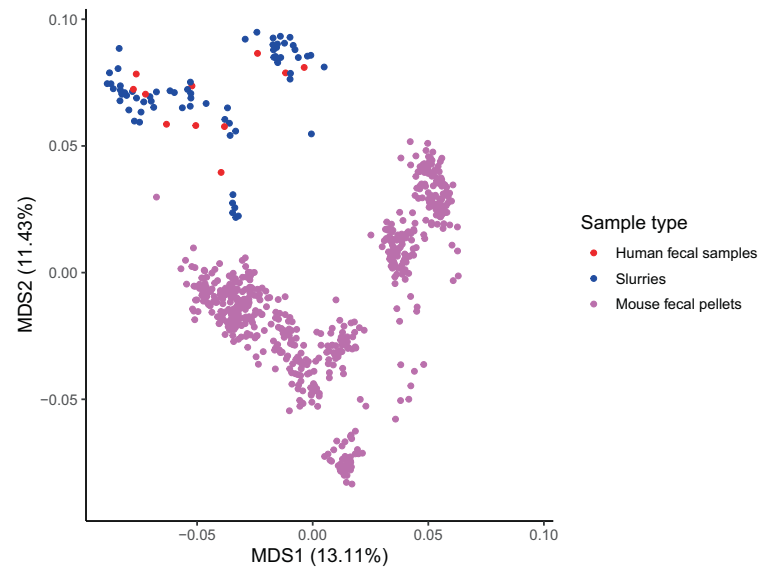

Fig. 1 Multidimensional scaling of microbial communities from human fecal samples, slurries, and mouse fecal pellets. a Multidimensional scaling of microbial composition from human fecal samples, slurries, and mouse fecal pellets color coded by sample type. b Multidimensional scaling of microbial composition from human

(Supplementary Fig. 4). In addition, the clustering patterns were dependent on the identity of the human donors (Fig. 1b; Supplementary Fig. 3b for paired read analysis). Human fecal samples and slurries explained $69.9 \%$ and $80.61 \%$ of variance, respectively, in mouse fecal microbiotas (ADONIS test, $p$ value $=0.001$ ). Our data are therefore consistent with previous studies reporting that the input of the human microbial community has a profound impact on the resulting microbial community in the colonized rodent [11, 15-17].

\section{Slurries adequately represent microbial communities in human fecal samples}

To determine the retention of gut microbiota when preparing slurries from human fecal samples, we calculated the presence of unique SVs in both human fecal samples $(n=$ 11 ) and the corresponding slurries $(n=63)$. The number of human fecal samples and slurries is based on the number of available samples with 16S rRNA gene sequencing data. A non-paired analysis (i.e., SVs were detected in at least 1 human fecal sample and 1 slurry) showed that $88.39 \%$ of SVs were shared between human fecal samples and slurries (Fig. 2a; Supplementary Fig. 5a for paired read analysis), which was not influenced by sequence depth (Supplementary Figs. 6, 7a, c) or filtering threshold (Supplementary Fig. 8a). However, a paired analysis (i.e., SVs must be present in the human fecal sample and its corresponding slurry) showed that on average $71.85 \pm 7.64 \%$ of SVs were shared between a human fecal sample-slurry pair with $13.88 \pm 9.78 \%$ of SVs found only in the slurry and $14.27 \pm$ $9.59 \%$ of SVs found only in the human fecal sample (Fig. 2b; Supplementary Fig. 5b for paired read analysis).
B

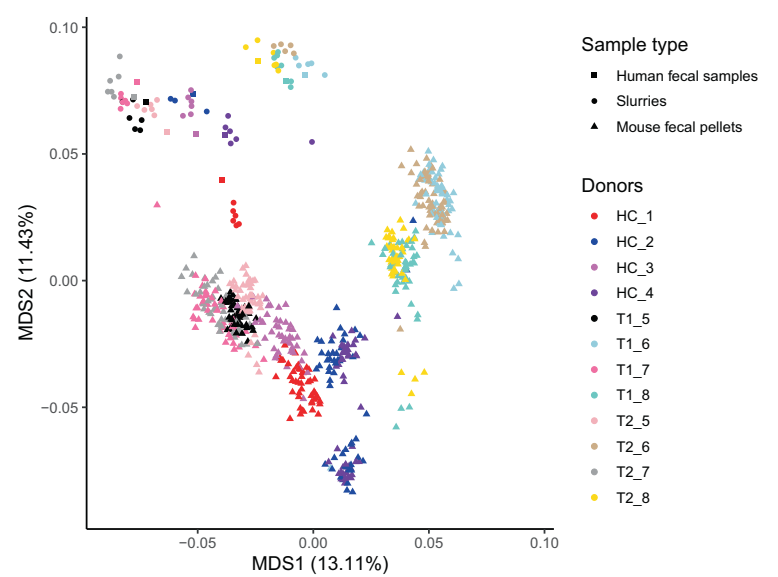

fecal samples, slurries, and mouse fecal pellets color coded by human donors. HC healthy control, T1 patient with anorexia nervosa before clinical renourishment, T2 patient with anorexia nervosa after clinical renourishment, MDS multidimensional scaling. Each number in the Donors legend identifies a unique donor.

As predicted, the frequency of SVs shared between human fecal sample-slurry pairs was strongly associated with the rank abundance of each SV, with more abundant SVs in human fecal samples having a greater probability of being present in the respective slurries (Fig. 2c; Supplementary Fig. 5c for paired read analysis). However, SVs that were detected only in human fecal samples or only in slurries did not have an obvious relationship to the overall rank abundance of the SVs in the human fecal samples (Fig. 2d, e; Supplementary Fig. 5d, e for paired read analysis). Overall, these data demonstrate that relatively abundant microorganisms are successfully retained during preparation of slurries from human fecal samples.

In addition to investigating whether SVs were observed in paired samples, we directly compared the relative abundance of all 279 SVs for each of the 63 human fecal sampleslurry pairs. Reassuringly, there was a strong correlation between relative abundances of SVs detected in a human fecal sample and its paired slurry $(n=63$ human fecal sample-slurry pairs, Spearman's correlation coefficient $(\rho)$ $=0.77 \pm 0.15$ ), suggesting that community composition was strongly preserved between paired human feces and slurries (Fig. 3a, b; Supplementary Fig. 9; Supplementary Fig. 10a, b for paired read analysis).

\section{Mouse fecal pellets only partially represent the microbial communities from slurries}

Using the same approach as the human fecal sample-slurry analysis, we compared SVs in 135 slurry-mouse fecal pellet pairs. A non-paired analysis (i.e., SVs were present in at least 1 slurry and mouse fecal pellet) showed that $95.34 \%$ of the SVs were shared between slurries and mouse fecal 
A

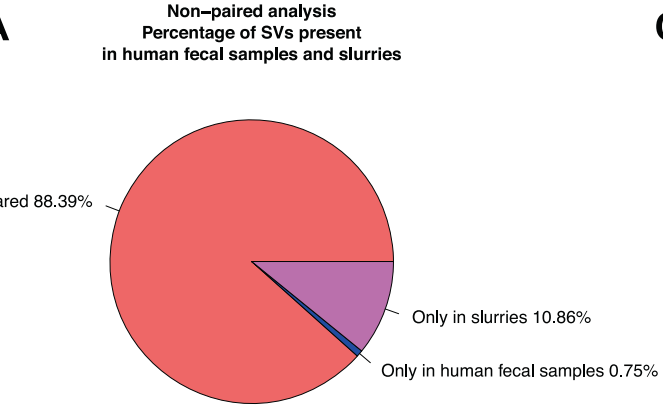

C

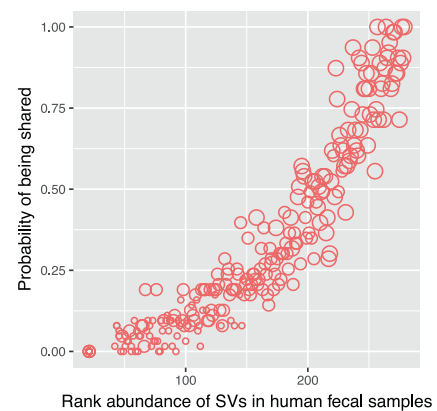

B

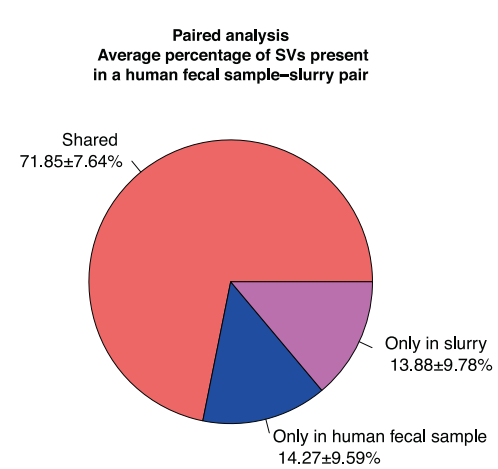

Fig. 2 Retention of SVs from human stool in slurries. a Percent of SVs that were detected in at least 1 human fecal sample and 1 slurry (shared: $88.39 \%$ ), detected in at least 1 human fecal sample but not in any slurry (only in human fecal samples: $0.75 \%$ ), detected in at least 1 slurry but not in any human fecal sample (only in slurries: $10.86 \%$ ). b Average percentages of SVs that were detected in a human fecal sample-slurry pair (shared: $71.85 \pm 7.64 \%$ ), detected in the human fecal sample but not the respective slurry (only in human fecal sample: $14.27 \pm 9.59 \%$ ), detected in the slurry but not in the respective human

pellets at week 1 post colonization. However, a paired analysis (i.e., SVs must be present in the slurry and the corresponding mouse fecal pellet) found that only $42.15 \pm$ 9.95\% SVs were shared between a slurry-mouse fecal pellet pair, with a significant number of SVs detected only in the slurry $(32.57 \pm 11.67 \%)$ or only in the mouse fecal pellet $(25.28 \pm 10.11 \%)$ at week 1 post colonization (Fig. $4 a, b)$. The percent of SVs shared between human slurries and mouse fecal samples was not influenced by sequencing depth (Supplementary Fig. 7b, d) or when pairing forward and reverse sequence reads (Supplementary Fig. 11a, b); however, a lower filtering threshold decreased the percent of SVs shared between human slurries and mouse fecal samples (Supplementary Fig. 8b). In addition, the percent of SVs shared between slurry-mouse fecal pellet pairs was not influenced by sex of the recipient mouse (Supplementary Fig. 12) and did not substantially change over the 4-week colonization period (Supplementary Figs. 13 and 14). Similar to findings from the human fecal sample-slurry analysis, SVs with a higher rank abundance in slurries were

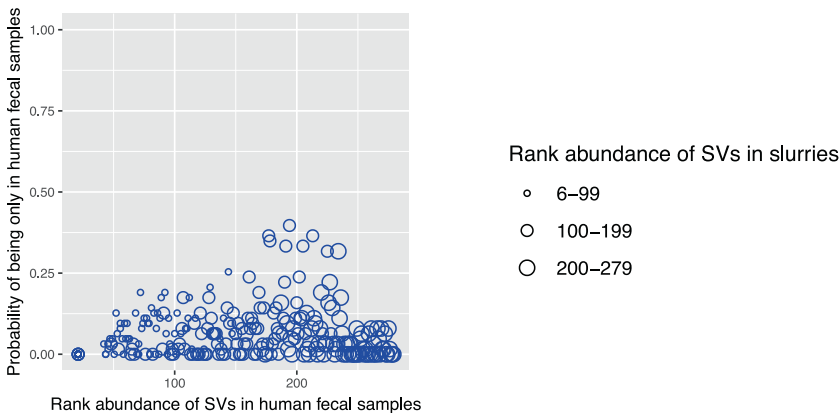

fecal sample (only in slurry: $13.88 \pm 9.78 \%$ ). Data are presented as mean \pm standard deviation. c Relationship between the probability of SVs being shared between human fecal sample-slurry pairs and rank abundance of SVs in human fecal samples. d Relationship between the probability of SVs being detected in slurries but not in their paired human fecal sample and rank abundance of SVs in human fecal samples. e Relationship between the probability of SVs detected in human fecal sample but not in their paired slurries and rank abundance of SVs in human fecal samples. SV sequence variant.

more likely to be successfully transferred into mice; however, 32 SVs with rank abundance of higher than 200 in slurries had a transfer rate less than $25 \%$ (dashed square in Fig. 4c, e; Supplementary Table 1; Supplementary Fig. 11c, e for paired read analysis). These SVs were significantly over- and under-represented by the Firmicutes and Bacteroidetes phyla, respectively, compared with the other highabundance taxa that had a transfer rate of more than $25 \%$ (Fisher's exact test, Firmicutes $p$ value $<0.001$; Bacteroidetes $p$ value $<0.01$ ). These SVs mostly belonged to the families Streptococcaceae, Lachnospiraceae, Ruminococcaceae, Veillonellaceae, Erysipelotrichaceae, Peptostreptococcaceae, and Family_XIII.

Surprisingly, $41 \mathrm{SVs}$ were found in more than 33 mouse fecal pellets but not in their paired slurries (dashed square in Fig. 4d; Supplementary Fig. 11d for paired read analysis). $90 \%$ of these 41 SVs were of low abundance in the entire set of slurries (rank abundance below 200) and belonged to the Firmicutes $(n=37)$, Bacteroidetes $(n=2)$, Proteobacteria $(n=1), \quad$ and Actinobacteria $(n=1)$ phyla, 
A

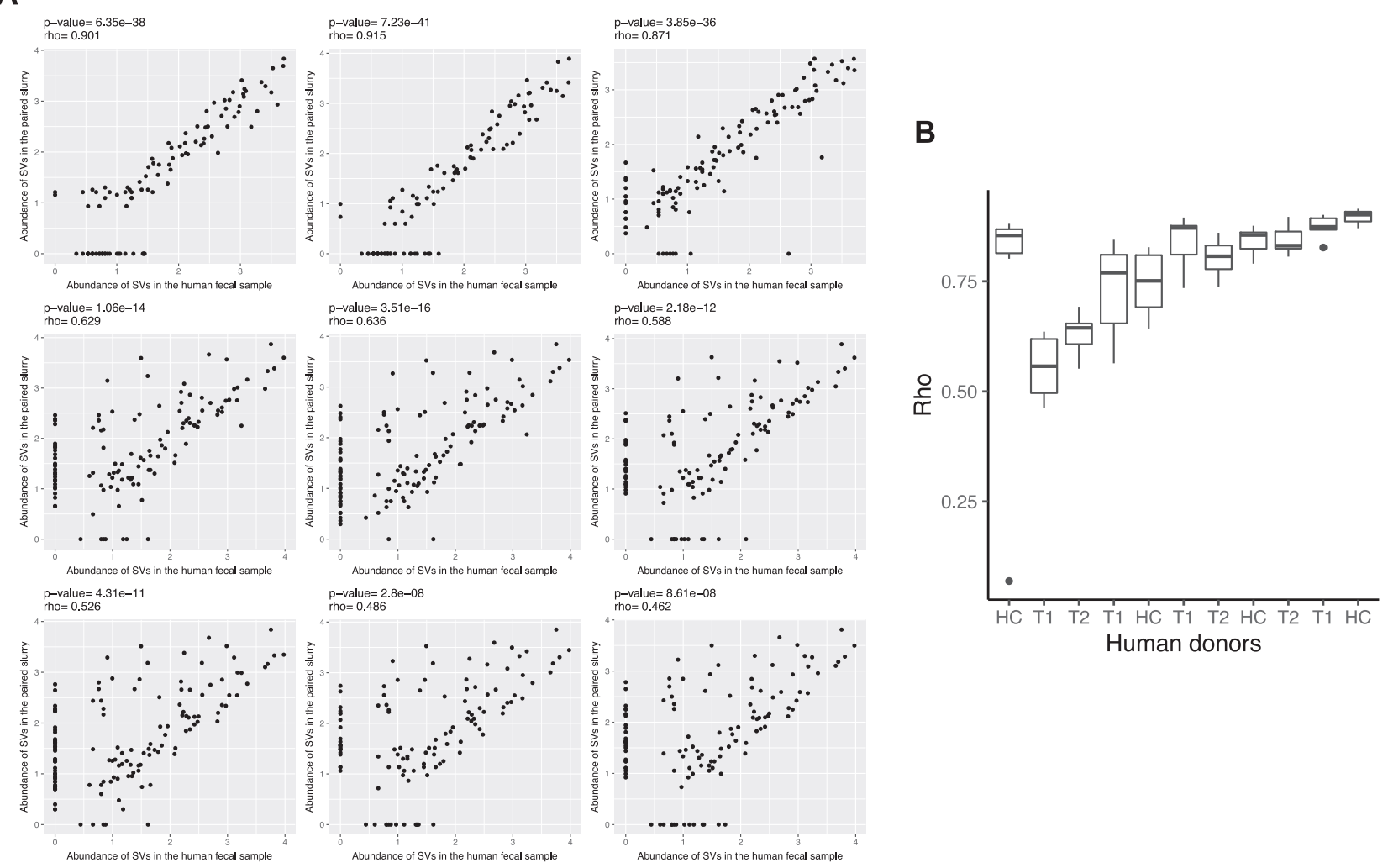

Fig. 3 Relationship between relative abundance of SVs in a human fecal sample-slurry pairs. a Spearman's rank-order correlations for each paired donor fecal-slurry sample $(n=63$ paired fecal-slurry samples). $p$ values were adjusted using the Benjamini-Hochberg $(\mathrm{BH})$ procedure. $x$ - and $y$-axes are $\log _{10}$-normalized relative abundance of SVs. Plots for all paired human fecal sample-slurry pairs are included

(Supplementary Table 2). At the family level, these SVs belonged to Bacteroidaceae, Coriobacteriaceae, Ruminococcaceae, Lachnospiraceae, Eubacteriaceae, Enterobacteriaceae, and Erysipelotrichaceae.

For each of the 135 mouse fecal pellet-slurry pairs, we directly compared the relative abundance of all 279 SVs in both environments. In contrast to the strong positive correlation between human fecal samples and slurries, the relative abundance of SVs was largely negatively correlated between slurries and mouse fecal pellets (mean $\rho=-0.20 \pm 0.12$ ) with 66 of the 135 pairs showing a statistically significant negative relationship at a 5\% FDR threshold (Fig. 5a, b, Supplementary Fig. 15; Supplementary Fig. 16a, b for paired read analysis). These negative correlations were not sex-dependent (Supplementary Fig. 17) and were largely due to the abundance of several human fecal taxa being poorly represented in mouse fecal pellets or, conversely, taxa enriched in mouse fecal pellets that were not detected in the paired slurries. The associations between human slurries and mouse fecal microbial communities remained the same at weeks 1, 2, 3, and 4 post colonization (Supplementary Fig. 18a). in Supplementary Fig. 9. b Boxplots showing Spearman correlation coefficients (rho: $\rho$ ) between human fecal sample-slurry pairs for each donor ( $n=3-7$ human fecal sample-slurry pairs per donor). HC healthy control, T1 patient with anorexia nervosa before clinical renourishment, T2 patient with anorexia nervosa after clinical renourishment, $\mathrm{SV}$ sequence variant.

\section{Most SVs in mouse fecal pellets show modest positive correlations with paired human fecal microbiota communities}

Up to this point, our analyses compared the relative abundance of all SVs in human feces, slurries, and mouse fecal pellets for each paired sample (63 human fecal sample-slurry pairs and 135 slurry-mouse fecal pellet pairs). In order to better estimate the transfer efficiency for each individual SV, we determined the association between the relative abundance of each of the $279 \mathrm{SVs}$ across all 135 slurry-mouse fecal pellet pairs. Using this approach, we found that $69 \%$ of SVs were positively correlated between slurries and mouse fecal pellets at week 4 post colonization at 5\% FDR (range of significantly positive $\rho=0.18-0.99$, Table 2 , Supplementary Table 3, Supplementary Fig. 18b, and Supplementary Fig. 19). These mostly modest correlations between slurries and mouse fecal pellets were not strong enough to result in similar overall microbial community composition between paired slurries and mouse fecal pellets, as shown in Fig. 5. 


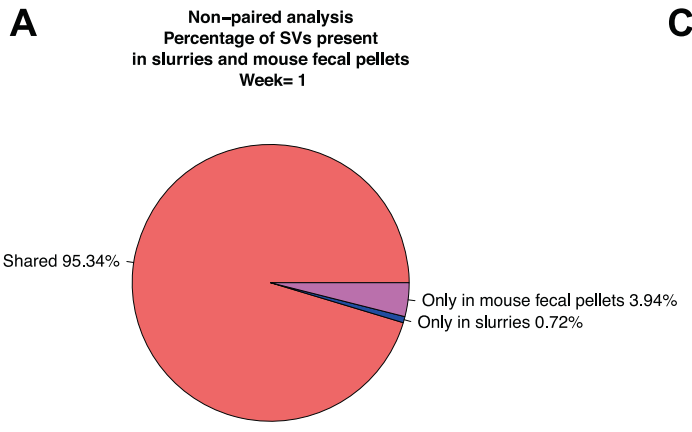

B

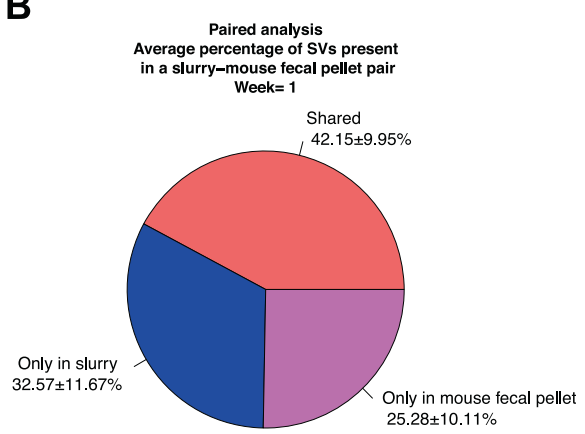

Fig. 4 Retention of SVs from slurries in recipient mice. a Percent of $\mathrm{SVs}$ that were detected in at least 1 slurry and 1 mouse fecal pellet (shared: $95.34 \%$ ), detected in at least 1 slurry but not in any mouse fecal pellet (only in slurries: $0.72 \%$ ), detected in at least in 1 mouse fecal pellets but not in any slurry (only in mouse fecal pellets: $3.94 \%$ ) 1 week after colonization. b Average percentages of SVs that were detected in a slurry-mouse fecal pellet pair (shared: $42.15 \pm 9.95 \%$ ), detected in the slurry but not in the respective mouse fecal pellet (only in slurry: $32.57 \pm 11.67 \%$ ), detected in the mouse fecal pellet but not in the respective slurry (only in mouse fecal pellet: $25.28 \pm 10.11 \%$ )

\section{Abundant members of Firmicutes fail to reliably transfer from slurries to mice}

To compare transfer efficiency of different taxa from slurries to GF mice, we compared Spearman correlation coefficients $(\rho)$ of SVs between different taxonomic classifications (Fig. 6a, Supplementary Fig. 20a-d). SVs belonging to the Bacteroidia class $(\rho=0.65 \pm 0.25)$ established more efficiently in mice compared with other taxonomic classes, such as Clostridia $(\rho=0.30 \pm 0.27)$ and Erysipelotrichia $(\rho=0.28 \pm 0.31)$ at week 4 post colonization (Supplementary Fig. 20b). At the family level, Bacteroidaceae $(\rho=0.66 \pm 0.28)$, Porphyromonadaceae $(\rho=$ $0.70 \pm 0.22)$, and Prevotellaceae $(\rho=0.76 \pm 0.07)$ had significantly greater Spearman correlation coefficients compared with Erysipelotrichaceae $(\rho=0.28 \pm 0.31)$, Lachnospiraceae $(\rho=0.26 \pm 0.27)$, and Ruminococcaceae $(\rho=0.31 \pm 0.27)$ at week 4 post colonization (Supplementary Fig. 20d). Consistent with previous results [11, 15-17], these data suggest that taxa belonging to Bacteroidetes have
D

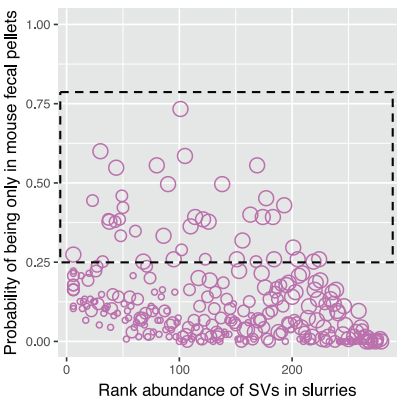

Rank abundance of SVs in mouse fecal pellets

- 1-99

○ $100-199$

○ 200-279

1 week after colonization. Data are presented as mean \pm standard deviation. c Relationship between the probability of SVs being shared between slurry-mouse fecal pellet pairs and rank abundance of SVs in slurries 1 week after colonization. d Relationship between the probability of SVs detected in mouse fecal pellets but not in their paired slurries and rank abundance of SVs in slurries 1 week after colonization. e Relationship between the probability of SVs detected in slurries but not in their paired mouse fecal pellets and rank abundance of SVs in slurries 1 week after colonization. SV sequence variant.

greater transfer efficiencies from human fecal samples to mice compared with members of the Firmicutes phylum.

As we observed a stronger human-mouse correlation for SVs belonging to Bacteroidetes compared with Firmicutes, we next compared the Firmicutes to Bacteroidetes ratio between human feces, slurries, and mouse fecal pellets. Mouse fecal pellets had the lowest Firmicutes to Bacteroidetes ratio $\left(\log _{10}\right.$ Firmicutes:Bacteroidetes $=0.32 \pm 0.26$, Fig. 6b), whereas human fecal samples had the highest Firmicutes to Bacteroidetes ratio ( $\log _{10}$ Firmicutes:Bacteroidetes $=2.05 \pm 0.66$, Fig. $6 \mathrm{~b}$ ), further supporting that Bacteroidetes originating from human fecal samples colonize GF mice more efficiently than Firmicutes.

\section{Discussion}

A major challenge for current intestinal microbiota research is moving from observational to mechanistic studies that reveal how members of these complex microbial 
A
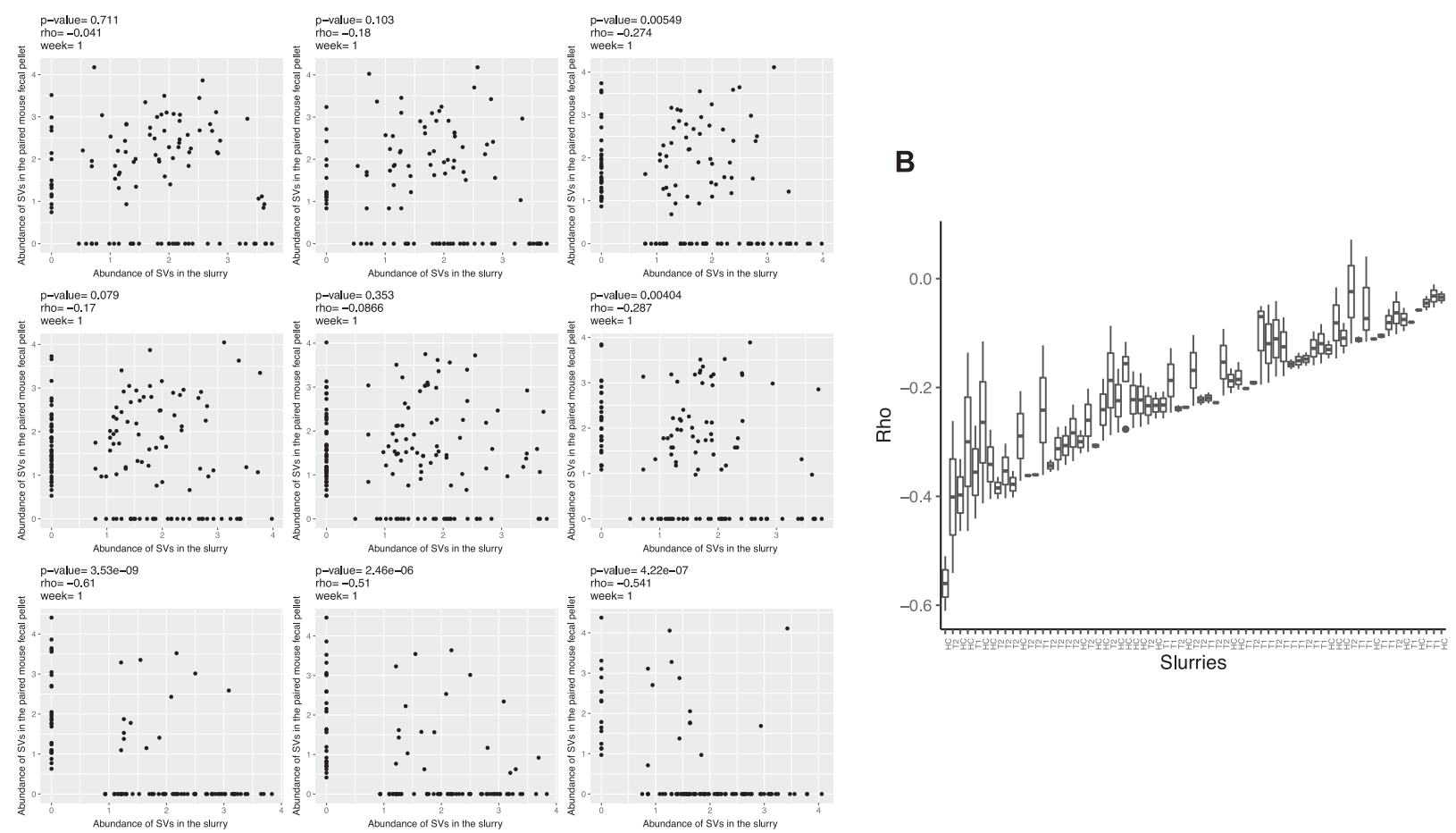

Fig. 5 Relationship between relative abundances of SVs in a slurryincluded in Supplementary Fig. 15. b Boxplots showing Spearman cormouse fecal pellet pairs 1 week after colonization. a Spearman's rank-order correlations for each slurry-mouse fecal pellet pair $(n=135$ slurry-mouse fecal pellet pairs). $p$ values were adjusted using the Benjamini-Hochberg (BH) procedure. $x$ - and $y$-axes are $\log _{10}$-normalized relative abundance of SVs. Plots for all slurry-mouse fecal pellet pairs are relation coefficients (rho: $\rho$ ) between slurry-mouse fecal pellet pairs for each slurry 1 week after colonization $(n=1-4$ paired slurry-mouse fecal pellet samples per slurry). HC healthy control, T1 patient with anorexia nervosa before clinical renourishment, $\mathrm{T} 2$ patient with anorexia nervosa after clinical renourishment, SV sequence variant.

Table 2 Representative SVs that were efficiently transferred from slurries to recipient mice 4 weeks after colonization.

\begin{tabular}{|c|c|c|c|c|c|}
\hline Sequence variant & Rho coefficient & $p$ value & Phylum & Family & Genus \\
\hline SV82 & 0.99 & $5.20 \mathrm{E}-110$ & Bacteroidetes & Porphyromonadaceae & Parabacteroides \\
\hline SV121 & 0.99 & $5.02 \mathrm{E}-104$ & Firmicutes & Ruminococcaceae & Ruminococcaceae_UCG-009 \\
\hline SV331 & 0.98 & $2.72 \mathrm{E}-94$ & Firmicutes & Lachnospiraceae & Lactonifactor \\
\hline SV405 & 0.96 & $9.54 \mathrm{E}-76$ & Bacteroidetes & Porphyromonadaceae & NA \\
\hline SV66 & 0.96 & $1.78 \mathrm{E}-73$ & Bacteroidetes & Bacteroidaceae & Bacteroides \\
\hline SV446 & 0.93 & $4.57 \mathrm{E}-57$ & Firmicutes & Ruminococcaceae & Anaerofilum \\
\hline SV30 & 0.93 & $3.98 \mathrm{E}-56$ & Bacteroidetes & Bacteroidaceae & Bacteroides \\
\hline SV277 & 0.92 & $2.28 \mathrm{E}-53$ & Actinobacteria & Coriobacteriaceae & NA \\
\hline SV100 & 0.92 & $2.28 \mathrm{E}-53$ & Bacteroidetes & Bacteroidaceae & Bacteroides \\
\hline SV109 & 0.91 & $5.21 \mathrm{E}-51$ & Bacteroidetes & Bacteroidaceae & Bacteroides \\
\hline SV127 & 0.91 & $6.48 \mathrm{E}-51$ & Firmicutes & Erysipelotrichaceae & Dielma \\
\hline SV81 & 0.90 & $1.00 \mathrm{E}-48$ & Bacteroidetes & Bacteroidaceae & Bacteroides \\
\hline SV274 & 0.89 & $5.68 \mathrm{E}-47$ & Proteobacteria & Desulfovibrionaceae & Desulfovibrio \\
\hline SV578 & 0.88 & $5.41 \mathrm{E}-44$ & Firmicutes & Defluviitaleaceae & Defluviitaleaceae_UCG-011 \\
\hline SV87 & 0.87 & $1.21 \mathrm{E}-41$ & Bacteroidetes & Bacteroidaceae & Bacteroides \\
\hline SV271 & 0.85 & $2.89 \mathrm{E}-37$ & Bacteroidetes & Porphyromonadaceae & Butyricimonas \\
\hline SV75 & 0.84 & $1.88 \mathrm{E}-36$ & Bacteroidetes & Rikenellaceae & Alistipes \\
\hline SV154 & 0.83 & $4.65 \mathrm{E}-34$ & Firmicutes & Ruminococcaceae & NA \\
\hline SV10 & 0.82 & $1.82 \mathrm{E}-33$ & Bacteroidetes & Prevotellaceae & Prevotella_9 \\
\hline SV172 & 0.82 & $1.14 \mathrm{E}-32$ & Firmicutes & Ruminococcaceae & Anaerofilum \\
\hline SV201 & 0.82 & $1.83 \mathrm{E}-32$ & Firmicutes & Ruminococcaceae & Ruminiclostridium_9 \\
\hline SV74 & 0.82 & $2.63 \mathrm{E}-32$ & Bacteroidetes & Rikenellaceae & Alistipes \\
\hline SV46 & 0.81 & $1.67 \mathrm{E}-31$ & Firmicutes & Lachnospiraceae & Lachnoclostridium \\
\hline
\end{tabular}

Transfer efficiency is represented by significant (adjusted $p$ value $<0.05$ ) Spearman correlation coefficients (rho: $\rho$ ) for each SV across 135 slurrymouse fecal pellet pairs 4 weeks after colonization. This table shows the first $23 \mathrm{SVs}$ with the highest transfer efficiency (rho coefficients $>0.80$ ). Other SVs with significant transfer efficiency are included in Supplementary Table 3. SV sequence variant, NA unclassified. 
A

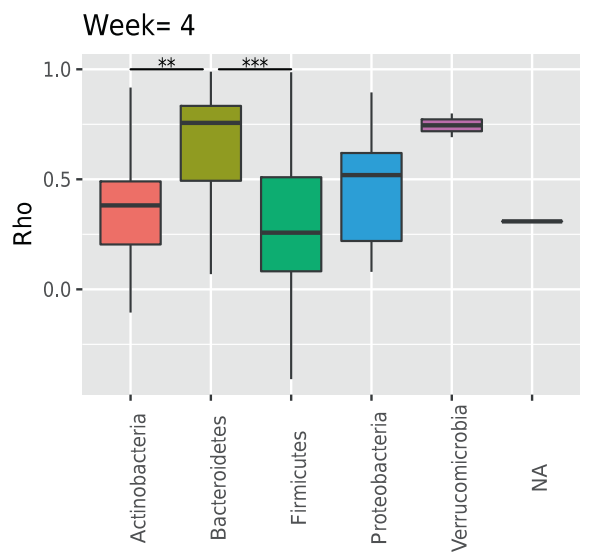

Fig. 6 Transfer efficiency across different bacterial taxa. a Boxplots showing transfer efficiency of SVs in slurries to recipient GF mice at the phylum level 4 weeks after colonization. Transfer efficiency is represented by the Spearman correlation coefficients (rho: $\rho$ ) for each SV across slurry-mouse fecal pellet pairs. Number of SVs in each phylum:

communities impact host physiology. GF mice colonized with fecal microbiotas remain one of the most appealing models to determine the impact of a disease-associated gut microbiota on the host. As part of our ongoing research interested in understanding the link between the human gut microbiota and its influence on metabolic aspects of AN we were motivated to investigate the efficiency of transplanting human fecal microbiotas into GF mice. Using a large sample size of gnotobiotic mice that resulted from these studies, we here report the results of identifying SVs from high-throughput $16 \mathrm{~S}$ rRNA gene sequencing to generate a high-resolution picture of the transfer efficiency of human fecal microbial communities into GF mice. Consistent with previous studies, we found that the introduction of human fecal microbiotas into a GF mouse results in distinct clustering patterns that are dependent on the identity of the human donor. Our findings also demonstrated that slurries prepared from human fecal samples adequately reflected the donor source microbiota. Conversely, on average, only $42 \%$ of SVs transferred from slurries to the corresponding GF mice, and the relative abundances of several human fecal taxa in the slurries were poorly represented in the mouse fecal pellets, and vice versa. Indeed, the presence of a few high-abundance taxa in the slurries that were absent in the mice led to weak, but statistically significant, negative correlations in taxa for many of the paired samples between mice and their respective slurries. While untangling the underlying biological factors responsible for the observed poor transfer efficiency is beyond the scope of our study, possible explanations have been discussed in detail in a
B

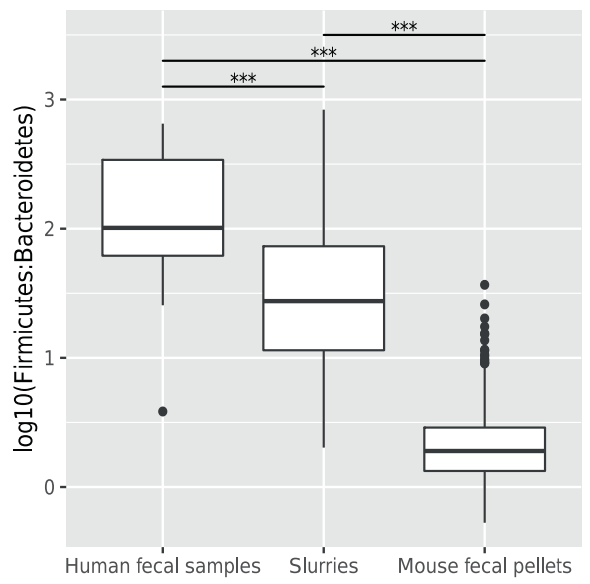

12 Actinobacteria, 39 Bacteroidetes, 211 Firmicutes, 7 Proteobacteria, 2 Verrucomicrobia, 1 Unclassified (NA). b $\log _{10}$ normalized Firmicutes to Bacteroidetes ratio in human fecal samples, slurries, and mouse fecal pellets. One-way ANOVA with a post-hoc Tukey's HSD, ***adjusted $p$ value $<0.001$, **adjusted $p$ value $<0.01$. SV sequence variant.

review by Arrieta et al. [20]. Some of these potential factors include the differences in GI anatomy and physiology between humans and mice, poor immune development in GF mice, and the drastic change in diet that microbial communities are exposed to when transplanted from humans to GF rodents.

Interestingly, consistent with other studies [11, 15-17], we found members of the Bacteroidetes phylum had greater colonization efficiency in GF mice than members of the Firmicutes phylum. We also found SVs that were detected only in mouse fecal pellets when compared with their corresponding slurries. There are two possible explanations for phenomenon; (i) these SVs may have been present in the slurries at abundances below 16S rRNA gene sequencing detection limits and then flourished upon entering a sterile GI tract, or (ii) these SVs may have been introduced into recipient mice from the environment. Given that the taxonomy of these SVs encompass typical enteric microbes and not microbes from human or environmental niches (e.g., microbes from the skin), we believe the former option to be more likely. It is more difficult to explain the origin of SVs found only in slurries and not present in their corresponding human fecal samples $(13.88 \pm 9.78 \%$ of all SVs in human fecal sample-slurry pairs). It is possible that these SVs are a result of environmental contamination as slurries were prepared in a clean, but non-sterile, anaerobic chamber. Given the importance of mirroring the composition of donor gut microbiotas in corresponding mouse recipients, reporting a standardized transfer rate in current and future GF colonization studies is critical. Transfer efficiency is 
frequently reported by examining the fraction of operational taxonomic units or SVs present in the human donor samples that is also present in any of the mouse samples. Our results argue that this "unpaired" approach overstates the true transfer efficiency and should be avoided. We propose an alternative method to calculate transfer efficiencies between paired samples (i.e., human donor and corresponding mouse recipient samples) to more accurately reflect the percent of $\mathrm{SVs}$, and their relative abundances, that successfully engraft in the recipient mouse.

This study also had limitations that should be noted. Importantly, we calculated transfer efficiencies using highthroughput sequencing of the 16S rRNA gene and therefore cannot differentiate between viable and non-viable microbes in the human fecal samples. Therefore, it is possible that our calculations have underestimated the actual rate of transfer efficiency as non-viable bacteria will not colonize the GI tract of the recipient mouse. Secondly, mice in this study were singly housed in ventilated cage racks in an SPF facility, rather than in a gnotobiotic isolator; however, a study using mice colonized with human fecal microbiotas and housed in gnotobiotic isolators reported a very similar transfer efficiency $(45 \%$ of genera and $20 \%$ of operational taxonomic units between human donor and mouse recipient) [12]. Nevertheless, animal housing and husbandry facilities are environmental factors that influence the murine gut microbiota and may also impact the transfer efficiency in gnotobiotic mouse models [33]. Thirdly, this study profiled the microbial communities using fecal pellets rather than cecal contents or mucosal tissue from the small and large intestines. We preferentially chose to analyze fecal pellets in order to investigate the effect of time on the rodent microbial communities; however, the transfer efficiency of microbial communities could differ if the same analysis were to be carried out using samples from a potentially more physiologically relevant site, such as the cecum or intestinal mucosal tissue. Fourthly, although our approach for collecting human fecal samples is consistent with methods used in multiple clinical studies, the samples we assembled were held at $4{ }^{\circ} \mathrm{C}$ for up to $24 \mathrm{~h}$ during transportation. While it is certainly possible that this storage at $4{ }^{\circ} \mathrm{C}$ impacted our results, it has been reported by multiple groups that transporting fecal samples for up to $48 \mathrm{~h}$ at $4{ }^{\circ} \mathrm{C}$ has a negligible impact on microbial communities [34-36]. Finally, although we use an established approach for characterizing complex microbial communities (16S rRNA gene sequencing), known PCR and sequencing biases are inherently embedded in this method [37, 38].

This study sheds light on an inherent limitation that exists when human fecal microbiotas are transplanted into GF mice. Notably, we identify a number of specific taxa that match their abundances in the donor sample when transplanted into GF mice. These taxa may be of clinical relevance and therefore valuable in the design of future studies using human fecal microbiotas and GF mice. In addition, whole-genome and transcriptome sequencing studies have the potential to help determine whether fecal microbiotas are functionally altered when transplanted into GF mice. It may also be worth performing similar compositional and functional studies in other gnotobiotic mammal models, such as pigs, that share more anatomical features to humans and could, therefore, provide an environmental niche that is more accommodating to the introduction of human microbiotas.

Acknowledgements The authors thank the nurses in the UNC Center of Excellence for Eating Disorders for their invaluable assistance collecting fecal samples from patients, Eun Young Huh and Yesel Trillo-Ordonez for providing technical assistance, the National Gnotobiotic Rodent Resource Center (NIH P30DK034987) for providing the germ-free mice, the UNC-Chapel Hill High-Throughput Sequencing Facility for performing the high-throughput sequencing, and the Lineberger Animal Studies Core (supported in part by an NCI Center Core Support Grant (CA16086)) for their assistance with rodent procedures. Finally, this study was supported by NIDDK grant P30DK056350 to the UNC Nutrition Obesity Research Center.

Funding This work was funded by the National Institute of Mental Health (R01 MH105684: PI Carroll). CMB acknowledges funding from the Swedish Research Council (Vetenskapsrådet, award: 5382013-8864).

\section{Compliance with ethical standards}

Conflict of interest CMB has served on advisory boards for Shire and receives royalties from Pearson. IMC and AAF have previously served as consultants for Salix Pharmaceuticals.

Publisher's note Springer Nature remains neutral with regard to jurisdictional claims in published maps and institutional affiliations.

\section{References}

1. Backhed F, Ding H, Wang T, Hooper LV, Koh GY, Nagy A, et al. The gut microbiota as an environmental factor that regulates fat storage. Proc Natl Acad Sci USA. 2004;101:15718-23.

2. Ley RE, Turnbaugh PJ, Klein S, Gordon JI. Microbial ecology: human gut microbes associated with obesity. Nature. 2006;444:1022-3.

3. Turnbaugh PJ, Backhed F, Fulton L, Gordon JI. Diet-induced obesity is linked to marked but reversible alterations in the mouse distal gut microbiome. Cell Host Microbe. 2008;3:213-23.

4. Karlsson FH, Tremaroli V, Nookaew I, Bergstrom G, Behre CJ, Fagerberg B, et al. Gut metagenome in European women with normal, impaired and diabetic glucose control. Nature. 2013;498:99-103.

5. Larsen N, Vogensen FK, van den Berg FW, Nielsen DS, Andreasen AS, Pedersen BK, et al. Gut microbiota in human adults with type 2 diabetes differs from non-diabetic adults. PLoS ONE. 2010;5:e9085. 
6. Wang T, Cai G, Qiu Y, Fei N, Zhang M, Pang X, et al. Structural segregation of gut microbiota between colorectal cancer patients and healthy volunteers. ISME J. 2012;6:320-9.

7. Franzosa EA, Sirota-Madi A, Avila-Pacheco J, Fornelos N, Haiser $\mathrm{HJ}$, Reinker S, et al. Gut microbiome structure and metabolic activity in inflammatory bowel disease. Nat Microbiol. 2019;4:293-305.

8. De Angelis M, Piccolo M, Vannini L, Siragusa S, De Giacomo A, Serrazzanetti DI, et al. Fecal microbiota and metabolome of children with autism and pervasive developmental disorder not otherwise specified. PLoS ONE. 2013;8:e76993.

9. Ridaura VK, Faith JJ, Rey FE, Cheng J, Duncan AE, Kau AL, et al. Gut microbiota from twins discordant for obesity modulate metabolism in mice. Science. 2013;341:1241214-10.

10. Turnbaugh PJ, Ley RE, Mahowald MA, Magrini V, Mardis ER, Gordon JI. An obesity-associated gut microbiome with increased capacity for energy harvest. Nature. 2006;444:1027-31.

11. Hintze KJ, Cox JE, Rompato G, Benninghoff AD, Ward RE, Broadbent $J$, et al. Broad scope method for creating humanized animal models for animal health and disease research through antibiotic treatment and human fecal transfer. Gut Microbes. 2014;5:183-91.

12. Zhang L, Bahl MI, Roager HM, Fonvig CE, Hellgren LI, Frandsen HL, et al. Environmental spread of microbes impacts the development of metabolic phenotypes in mice transplanted with microbial communities from humans. ISME J. 2017;11:676-90.

13. Fouladi F, Brooks AE, Fodor AA, Carroll IM, Bulik-Sullivan EC, Tsilimigras MCB, et al. The role of the gut microbiota in sustained weight loss following Roux-en-Y gastric bypass surgery. Obes Surg. 2019;29:1259-67.

14. Tremaroli V, Karlsson F, Werling M, Ståhlman M, KovatchevaDatchary P, Olbers T, et al. Roux-en-Y gastric bypass and vertical banded gastroplasty induce long-term changes on the human gut microbiome contributing to fat mass regulation. Cell Metab. 2015;22:228-38.

15. Staley C, Kaiser T, Beura LK, Hamilton MJ, Weingarden AR, Bobr A, et al. Stable engraftment of human microbiota into mice with a single oral gavage following antibiotic conditioning. Microbiome. 2017;5:87.

16. Wos-Oxley M, Bleich A, Oxley AP, Kahl S, Janus LM, Smoczek A, et al. Comparative evaluation of establishing a human gut microbial community within rodent models. Gut Microbes. 2012;3:234-49.

17. Chung H, Pamp SJ, Hill JA, Surana NK, Edelman SM, Troy EB, et al. Gut immune maturation depends on colonization with a hostspecific microbiota. Cell. 2012;149:1578-93.

18. Turnbaugh PJ, Ridaura VK, Faith JJ, Rey FE, Knight R, Gordon JI. The effect of diet on the human gut microbiome: a metagenomic analysis in humanized gnotobiotic mice. Sci Transl Med. 2009; 1:6ra14

19. Rawls JF, Mahowald MA, Ley RE, Gordon JI. Reciprocal gut microbiota transplants from zebrafish and mice to germ-free recipients reveal host habitat selection. Cell. 2006;127:423-33.

20. Arrieta MC, Walter J, Finlay BB. Human microbiota-associated mice: a model with challenges. Cell Host Microbe. 2016; 19:575-8.

21. Campbell JH, Foster CM, Vishnivetskaya T, Campbell AG, Yang ZK, Wymore A, et al. Host genetic and environmental effects on mouse intestinal microbiota. ISME J. 2012;6:2033-44.

22. McCafferty J, Muhlbauer M, Gharaibeh RZ, Arthur JC, PerezChanona E, Sha W, et al. Stochastic changes over time and not founder effects drive cage effects in microbial community assembly in a mouse model. ISME J. 2013;7:2116-25.

23. Le Roy T, Debedat J, Marquet F, Da-Cunha C, Ichou F, GuerreMillo M, et al. Comparative evaluation of microbiota engraftment following fecal microbiota transfer in mice models: age, kinetic and microbial status matter. Front Microbiol. 2018;9:3289.

24. Callahan BJ, McMurdie PJ, Rosen MJ, Han AW, Johnson AJ, Holmes SP. DADA2: high-resolution sample inference from Illumina amplicon data. Nat Methods. 2016;13:581-3.

25. Edgar RC UNOISE2: improved error-correction for Illumina $16 \mathrm{~S}$ and ITS amplicon sequencing. bioRxiv. 2016. https://doi.org/10. $1101 / 081257$.

26. Amir A, McDonald D, Navas-Molina JA, Kopylova E, Morton JT, Zech Xu Z, et al. Deblur rapidly resolves single-nucleotide community sequence patterns. mSystems. 2017;2:e00191-16.

27. David LA, Maurice CF, Carmody RN, Gootenberg DB, Button JE, Wolfe BE, et al. Diet rapidly and reproducibly alters the human gut microbiome. Nature. 2014;505:559-63.

28. Feeding and eating disorders. In: AE Kazdin, editor. Diagnostic and statistical manual of mental disorders, 5th edn. Washington DC; American Psychiatric Association; 2013.

29. Kleiman SC, Bulik-Sullivan EC, Glenny EM, Zerwas SC, Huh EY, Tsilimigras MCB, et al. The gut-brain axis in healthy females: lack of significant association between microbial composition and diversity with psychiatric measures. PLoS ONE. 2017;12: $\mathrm{e} 0170208$.

30. Kleiman SC, Watson HJ, Bulik-Sullivan EC, Huh EY, Tarantino LM, Bulik CM, et al. The intestinal microbiota in acute anorexia nervosa and during renourishment: relationship to depression, anxiety, and eating disorder psychopathology. Psychosom Med. 2015;77:969-81.

31. Carroll IM, Ringel-Kulka T, Keku TO, Chang Y-H, Packey CD, Sartor RB, et al. Molecular analysis of the luminal- and mucosalassociated intestinal microbiota in diarrhea-predominant irritable bowel syndrome. Am J Physiol Gastrointest Liver Physiol. 2011;301:G799-807.

32. Kleiman SC, Glenny EM, Bulik-Sullivan EC, Huh EY, Tsilimigras $\mathrm{MCB}$, Fodor AA, et al. Daily changes in composition and diversity of the intestinal microbiota in patients with anorexia nervosa: a series of three cases. Eur Eat Disord Rev. 2017;25:423-7.

33. Parker KD, Albeke SE, Gigley JP, Goldstein AM, Ward NL. Microbiome composition in both wild-type and disease model mice is heavily influenced by mouse facility. Front Microbiol. 2018;9:1598.

34. Choo JM, Leong LE, Rogers GB. Sample storage conditions significantly influence faecal microbiome profiles. Sci Rep. 2015;5:16350.

35. Kim D, Hofstaedter CE, Zhao C, Mattei L, Tanes C, Clarke E, et al. Optimizing methods and dodging pitfalls in microbiome research. Microbiome. 2017;5:52.

36. Tedjo DI, Jonkers DM, Savelkoul PH, Masclee AA, van Best N, Pierik MJ, et al. The effect of sampling and storage on the fecal microbiota composition in healthy and diseased subjects. PLoS ONE. 2015;10:e126685.

37. Pollock J, Glendinning L, Wisedchanwet T, Watson M. The madness of microbiome: attempting to find consensus "best practice" for 16S microbiome studies. Appl Environ Microbiol. 2018;84;e02627-17.

38. McLaren MR, Willis AD, Callahan BJ. Consistent and correctable bias in metagenomic sequencing experiments. Elife. 2019;8: e46923. 